\title{
Cortical Wound Healing in the Amphibian Egg: An Electron Microscopical Study
}

\author{
JohN G. BLuEMINK \\ Hubrecht Laboratory, Uppsalalaan 1, Utrecht, The Netherlands \\ Received April 4, 1972
}

\begin{abstract}
Changes following injury of the animal pole cortex of fertilized uncleaved eggs of Xenopus laevis were studied with the electron microscope. In the course of the healing process the egg surface bordering the exovate protruding from a tear wound is thrown into folds. Pigment granules aggregate in the region below the edges of the damaged area. Concomitantly filament arrays come into being there. Fuzzy material is present in a diffuse and in a condensed form. It coats the membrane in the region of the surface folds as well as membrane surfaces inside the exovate. Parts of the exovate surface are in the form of so-called "crenelated layer." Probably this layer has a transitory sealing function and is homologous to the "new membrane" formed in wounded amoebae (18). The morphological alterations are interpreted as manifestations of membrane growth, active or passive contraction of filament arrays, and cytoplasmic coagulation. In this context Holtfreter's concept of a "surface coat" and his interpretation of cortical wound healing is reexamined.
\end{abstract}

In a well known paper entitled "Properties and functions of the surface coat in amphibian embryos," Holtfreter (9) first formulated his ideas about the existence of a socalled "surface coat." According to his original concept ${ }^{1}$, various important functions may be attributed to this coat, one of them being the wound healing capacity. Describing the structural features of the surface coat Holtfreter states (p. 262): "In amphibian eggs the surface layer is formed before, not after fertilization. It is not hyaline but contains small yolk granules and is in some regions characterized by a dense accumulation of spherical black granules"; (p. 263). "... fragments of the cortical membrane which have been isolated with glass needles always have particles of the underlying

1 In the context of this paper the term "surface coat" (s.c.) refers to the cell membrane proper and an underlying layer of peripheral cytoplasm that is a few microns thick. This conforms to H.'s first concept. Later, in an analysis of the process of gastrulation H. ascribed to the "s.c." a coordinating function at the supracellular level ( J. Exp. Zool. 94, 261, 1943). Accordingly H. illustrated (Fig. 1) the "s.c." as a supra-(= extra) cellular structural entity. In reality such an extracellular layer does not exist (Dollander, A.. Arch. Histol. Embryol., 44, 93, 1962). 
cytoplasm adhering to them." According to his original concept the surface coat is an elastic surface layer capable of expansion and contraction. Holtfreter also gives a detailed description of the process of cortical wound healing. His interpretation with regard to the mechanism of wound closure and the function of the surface coat can be summarized in the following quotation (p. 280): “"... the stretching of dye marks or of cells in the circumference of a wound is really due to active spreading of the coat and not, as might be assumed at a first glance, to being stretched by an actively constricting wound edge".

More recently Gingell (7) and Luckenbill (13) have each presented an electron microscopical study on the wound healing process in the amphibian cortex. Gingell worked with eggs of Xenopus laevis. Data derived from electron microscopy and from experimentally induced cortical contraction led him to propose that a calcium-activated contractile mechanism resides in the cytoplasmic region just beneath the plasma membrane.

Luckenbill (13) studied cortical wound healing in eggs of Ambystoma mexicanum. Ultrathin sections cut tangential to the wound surface show that dense material is present in a ring-shaped area, 4-8 $\mu \mathrm{m}$ wide, lying along the periphery of the wound. The dense material consists of fine filamentous elements and particles forming a meshwork. The author correlates the location of the ring-shaped area with the observation that the pigmented edge of the wound diminishes in diameter during closure. This leads her to compare the functioning of this area with that of a drawn purse string.

The two studies mentioned have not produced irrefutable evidence for the presence of aligned filaments. Recent increasing interest in the occurrence and function of cellular contractile systems makes it worthwhile to reinvestigate the phenomenon of surface repair. Since recent studies have put most emphasis on the involvement of a contractile system, it seems useful to recall Holtfreter's original interpretation and to reexamine the phenomenon, bearing both alternative interpretations in mind.

\section{MATERIALS AND METHODS}

Eggs of Xenopus laevis were obtained from hormonally stimulated couples reared in the laboratory. The jelly was removed from freshly laid eggs by placing them in a solution of papain and cysteine- $\mathrm{HCl}$ (adjusted to $\mathrm{pH} 7.8$ ) for 2 to 4 minutes (3). They were then thoroughly rinsed and kept in Niu-Twitty solution (15). The vitelline membrane was removed from fertilized uncleaved eggs with fine forceps. This was done in a culture dish with the bottom covered with $5 \%$ agar. Only eggs which were undamaged after the removal of the vitelline membrane were used for wound healing experiments. To make a wound, sharpened No. 5 steel forceps were used to pinch and tear off a tiny piece of the pigmented surface.

Eggs were fixed for electron microscopy at time intervals between 2 and 15 minutes after 
wounding. This was done by gently adding fixative to the culture dish. After half an hour the fluid was partly replaced by fresh fixative. This procedure was repeated two or three times. Eggs were fixed at room temperature, using two different aldehyde solutions as the primary fixative. Fixation solution A (method A) contained $1 \%$ acrolein and $2.5 \%$ glutaraldehyde with $1 \mathrm{mM} \mathrm{CaCl}$ in $0.067 \mathrm{M}$ cacodylate buffer (pH 7.4). Solution B (method B) contained $2.5 \%$ glutaraldehyde, $70 \mathrm{~m} M \mathrm{KCl}, 15 \mathrm{mM} \mathrm{NaCl}$, and $1 \mathrm{mM} \mathrm{CaCl} 2$ in $0.034 \mathrm{M} s$-collidine buffer ( $\mathrm{pH}$ 7.2). The fixation time varied between 8 and 20 hours. After the aldehyde fixation the eggs were rinsed and kept in the corresponding buffered salt solution. The eggs were cut to isolate the wound areas, which were then embedded in oriented position in $5 \%$ agar at $55^{\circ} \mathrm{C}$. The agar blocks were postfixed in a solution containing $1 \%$ osmium tetroxide in the corresponding buffered salt solution during 3.5 hours at room temperature. After osmium fixation the specimens were briefly rinsed again in the corresponding buffered salt solution and transferred to $70 \%$ Cellosolve containing $1 \%$ phosphotungstic acid. The blocks were kept in this solution for 1 hour at $4^{\circ} \mathrm{C}$. They were subsequently dehydrated in the cold in increasing concentrations of Cellosolve. The final $100 \%$ Cellosolve was replaced by propylene oxide (room temperature) prior to impregnation with Epon.

For embedding a modification of the Dow epoxy resin mixture as proposed originally by Lockwood (12) was used. The mixture contained 7.5 g DER 332, $2.7 \mathrm{~g}$ DER 732, $8.2 \mathrm{~g}$ DDSA, and $0.3 \mathrm{~g}$ DMP-30. The final impregnation was carried out by keeping the specimens for 1-2 hours in a freshly prepared mixture under vacuum. The casts were polymerized for 48 hours at $60^{\circ} \mathrm{C}$ at a relative humidity of $25 \%$. Sections of $1.5 \mu \mathrm{m}$ were cut with glass knives and used unstained for examination under phase contrast. Ultrathin sections were cut on a Reichert Om U2 ultramicrotome fitted with a diamond knife with an included angle of $48^{\circ}$. The thin sections collected on naked grids were stained during 1 or 2 minutes with lead citrate according to a method proposed by Venable and Coggeshall (20), and examined with a Zeiss electron microscope EM9 S2, using 30 or $50 \mu \mathrm{m}$ objective apertures.

A procedure originally elaborated by De Bruijn (4) was used to reduce the loss of lipid (method C). The aim of the method is to stabilize components such as phospholipids by a modified fixation and to reduce the loss of such material during the steps of dehydration and embedding. The idea of adding $\mathrm{K}_{3} \mathrm{Fe}(\mathrm{CN})_{6}$ and $\mathrm{CaCl}_{2}$ to the osmium fixative was developed empirically from the method of tricomplex stabilization of amphoteric phospholipids (5).

Eggs were fixed during 24 hours at room temperature in aldehyde solution A using freshly distilled acrolein. They were rinsed with buffer and stored overnight (in the dark and the cold) in 0.067 cacodylate buffer at $\mathrm{pH} 7.2$, containing $1 \mathrm{mM} \mathrm{CaCl}$. Postfixation was in $1 \% \mathrm{OsO}_{4}$ in $0.067 \mathrm{M}$ cacodylate buffer at $\mathrm{pH} 7.2$, containing $0.05 \mathrm{M} \mathrm{K}_{3} \mathrm{Fe}(\mathrm{CN})_{6}$ and $0.05 \mathrm{M}$ $\mathrm{CaCl}_{2}$, during 3.5 hours (in the dark and the cold). After osmium fixation the specimens were rinsed several times in cold $0.067 \mathrm{M}$ cacodylate buffer and dehydrated rapidly in increasing concentrations of cold acetone. The specimens were transferred from absolute acetone to a $3: 1$ acetone/Epon mixture (for 1 hour at room temperature), then to a $1: 1$ mixture (for 3 hours), and finally to a $1: 3$ mixture (for 12 hours). The final impregnation was for 6 hours in a freshly prepared mixture of $4.3 \mathrm{~g}$ Epon 812, $4.3 \mathrm{~g}$ DDSA, $1.4 \mathrm{~g}$ MNA, and $0.15 \mathrm{~g}$ DMP-30 (according to Elbers, personal communication). The polymerized blocks were relatively soft and had to be cut with glass knives. The thin sections were not sufficiently rigid to be examined without support. Therefore sections were collected on grids provided with a supporting film of Pioloform F (17). 
The undamaged egg surface

\section{RESULTS}

In ultrathin sections cut perpendicular to the cell membrane, the egg surface shows a corrugated profile (Fig. 1). The protruding parts have a broad base. They are delineated by a triple-layered membrane having a center-to-center black line spacing of about $5 \mathrm{~nm}$ (Fig. 3). Feltlike material forms a continuous layer (dense layer) underneath the cell membrane. It varies in thickness from 0.1 to $1 \mu \mathrm{m}$. The cytoplasm subjacent to the peripheral layer contains pigment granules and light vesicles or cisternae. These cell inclusions are particularly numerous here, while small yolk granules and lipid droplets which are also present are more numerous in the subperipheral cytoplasm. The cytoplasmic matrix mainly consists of ca. $30 \mathrm{~nm}$ particles, probably glycogen, but also contains ca. $16 \mathrm{~nm}$ particles, probably ribosomes.

\section{The isolated cortex}

Fertilized uncleaved Xenopus eggs devoid of their vitelline membrane were used for the isolation experiments. The operations were carried out in Niu-Twitty culture solution (15). In the animal pole region small fragments of the pigment-containing surface layer could be neatly prepared free. The consistency of the isolated layer is remarkable. It shows a tendency to crumple when left as an isolated piece in the culture solution. Therefore the isolated pieces were immediately transferred to the fixation solution, using a braking pipette. Properly fixed flat fragments were embedded in $5 \%$ agar at $55^{\circ} \mathrm{C}$ before being processed further.

Such a fragment of isolated egg surface is about $5 \mu \mathrm{m}$ thick (Fig. 2). The cell membrane delineates the fragment on the side showing a highly corrugated and continuous profile (Fig. 3). The cell membrane is underlaid by feltlike material forming an uneven but virtually continuous layer. Immediately subjacent are pigment granules, light vesicles, mitochondria, lipid droplets, and small yolk granules. On this side the fragment has an open structure. The minor cytoplasmic constituents forming the matrix have been dissolved out. Nevertheless, those cellular elements still present are so coherent that they form a structural entity of remarkable consistency. According to Holtfreter's original concept ( 9 ) manyfold functions may be attributed to this "surface coat," among others, the wound healing capacity.

Fra. 1. Fertilized uncleaved egg of Xenopus: profile of the animal pole surface. Dense layer (DL), lipid droplets (L), light vesicles (LV), mitochondria (M), and yolk granules (Y). Fixative $B$, $\times 11000$. FIG. 2. Isolated cortex. The closed surface (top) is corrugated and delineated by the plasma membrane (see also Fig. 3). The cytoplasmic side (bottom) is open. Fixative $B . \times 4000$.

FIG. 3. Isolated cortex. The plasma membrane is intact. Empty areas show that glycogen and ribosomal particles have been dissolved out. Fixative $B . \times 75000$. 

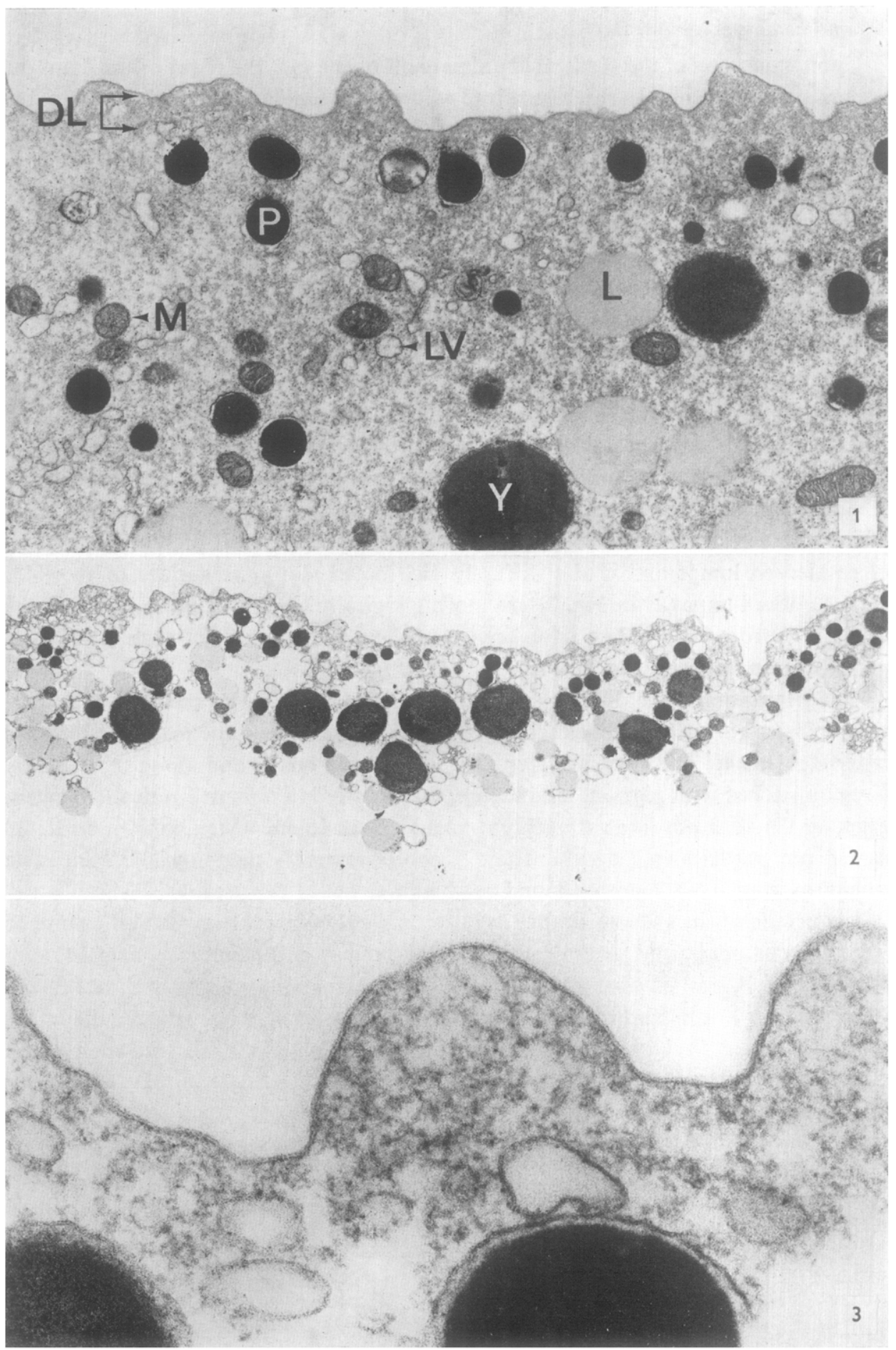


\section{Wound healing experiments}

Upon wounding of the pigmented animal half of the egg, the edges of the damaged area retract somewhat. In small puncture wounds the edges readily stick together, but in tear wounds the frayed edges smooth out to form a more or less circular opening. A little cytoplasm oozes out (exovate), forming a clot which adheres to the damaged area. This shows that cytoplasm once extruded coagulates rapidly. Pigment granules accumulate under the intact surface circumferential to the wound, whereas peripheral to this rim the cortex may become paler. As a result the wound after 4-6 minutes shows up as a ring of increased pigmentation (Fig. 4).

As seen in cross section the wound edge has a complex structure (Fig. 5). Peripheral to the damaged area the intact egg surface is smooth over a wide area. Here the number of pigment granules per unit area seems to be less than normal. Next to the wound the smooth surface abruptly passes into a narrow zone showing a highly convoluted surface. Slender surface folds are separated at their bases by cavities. In sections cut tangential to the wound area it can be shown that the surface is indeed convoluted (Fig. 6). The slender filopodia seen in Fig. 5 represent cross-sectioned surface folds which meander and branch. Extramembranous fuzzy material is present in the form of condensed lumps and is also diffusely distributed along the surface of the folds (Fig. 6). The lumps (as in Fig. 7) are morphologically similar to those found in the cleavage furrow, where they have been related to the presence of surface material possessing an adhesive function (1).

The cytoplasmic zone directly subjacent to the narrow, highly convoluted surface area is free of pigment and has an alveolar appearance due to the many cavities and cisternae present. The alveolar cytoplasm is bordered below and along the sides by a region packed with pigment granules (Fig. 5). Partly intermingled with the pigment granules 0.5 to $2 \mu \mathrm{m}$ large translucent vacuoles are found which contain scattered ca. $30 \mathrm{~nm}$ particles (glycogen?). These vacuoles resemble the so-called "plages de polyribosomes" $(19)$. They are found nowhere else but in this area.

The profile of the exovate surface looks crumpled. None of the exovates examined were left undamaged by the preparation procedure. A crenelated layer consisting of semicircular lumps of fibrillar material aligned along a triple-layered membrane (Figs. 8 and 9) forms part of the exovate surface in many eggs. In between the lumps the membrane is straight, while underneath a lump it bulges out into the open end of

FIG. 4. Phase contrast micrograph of an open cortical wound (top part of exovate damaged). Pigment granules have accumulated under the elevated wound edges. The intact egg surface is smooth (compare with Fig. 10). The framed area indicates the marginal zone shown in Fig. 5. Fixative $B, \times 640$.

FIG. 5. Open cortical wound: marginal zone. The smooth intact egg surface (left) abruptly passes into a narrow zone with a highly convoluted surface. Alveolar cytoplasm $(A P)$, vacuoles $(V)$, exovate $(E O)$. Small arrow: exovate surface layer. Fixative $B . \times 4400$. 


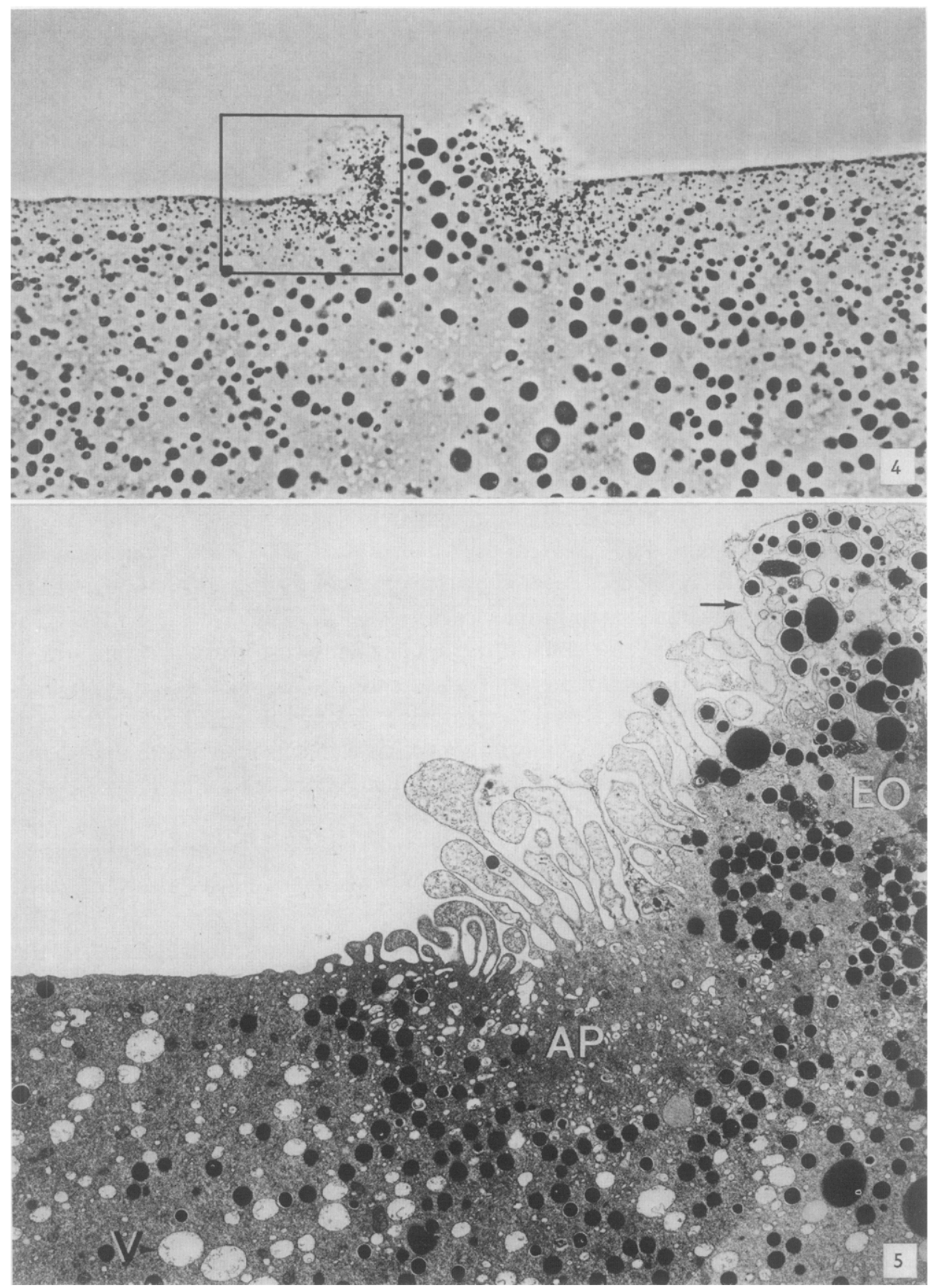


the semicircular structure (Fig. 9). The cytoplasm in the exovate contains cisternae with flocculent contents, lipid droplets, distorted mitochondria, partly degraded yolk granules, and pigment granules (Fig. 8). Many pigment granules lack an investing membrane, while others lie together and share a common envelope. Fuzzy material is present everywhere and coats all membrane surfaces.

When the exovate is removed during wound healing, the healing process is not stopped but some cytoplasm oozes out again at once. It shows up as a "rubbish heap" of membranes, cisternae, and other cell inclusions (not shown in the figures). Interesting are those peripheral parts where folded stretches of triple-layered membrane form the new outer surface of the exovate. In those places where membranes run parallel, amorphous material is sandwiched in between them in such a manner that the membranes give the impression of still forming a closed surface.

After the edges of a tear wound have changed into a slightly thickened ring, the ring starts to decrease in size. In the medium phase of healing extending from about 5-8 minutes after wounding, the smooth cell surface peripheral to the damaged area changes its profile (Fig. 10). The highly convoluted surface seen at the wound edge in an earlier phase (Figs. 4 and 5) expands. An area of ca. 100 to $200 \mu \mathrm{m}$ in diameter, circumferential to the wound, is covered by strongly folded surface (Figs. 10 and 11). Condensed fuzzy exudate is seen in the cavities (Fig. 12). The alveolar cytoplasm at the base of the folds next to the exovate contains dense cytoplasm in the form of conglomerates of fine, disperse material (Figs. 11 and 12). The bulk of such a conglomerate consists of particulate elements measuring 7-8 nm (Fig. 16), while the periphery shows up as a meshwork of short filamentous elements identical to that shown by Luckenbill (13, Fig. 5) in Ambystoma. Large conglomerates are about 1-2 $\mu \mathrm{m}$ in diameter. In the center, several of them have a dense core surrounded by a lighter halo (Fig. 16). Beside the large conglomerates there are smaller ones. The pigment granules in the cytoplasm beneath the wound are now evenly scattered, but still densely packed throughout the area (compare Figs. 10 and 4).

In order to visualize oriented filaments thin sections were cut tangential to the damaged surface. Bundles of filaments were found in tear wounds in the medium and late phases of healing. I have seen bundles like the one presented in Fig. 14 which were about 0.5 to $1 \mu \mathrm{m}$ wide and about $6 \mu \mathrm{m}$ long. The bundles are located in a region

FIG. 6. Marginal zone of an open wound, tangential section. The surface folds meander and branch. Lumps of exudate (arrows) adhere to the surface (see also Fig. 12). Fixative $B, \times 14500$.

FIG. 7 (inset). Lump of dense fibrous material which coats the tip of a surface fold. Fixative $A . \times 75900$. FIG. 8. Part of the crenelated layer seen on the exovate surface (arrows). The debris making up the exovate largely consists of membranous material. Fixative $B . \times 9000$.

FIG. 9. The crenelated layer consists of a triple-layered membrane showing outpocketings covered by a cap of dense fibrous material. On the inner side "droplets" of rather light electron density are vaguely visible (arrows). Fixative $B . \times 101200$. 


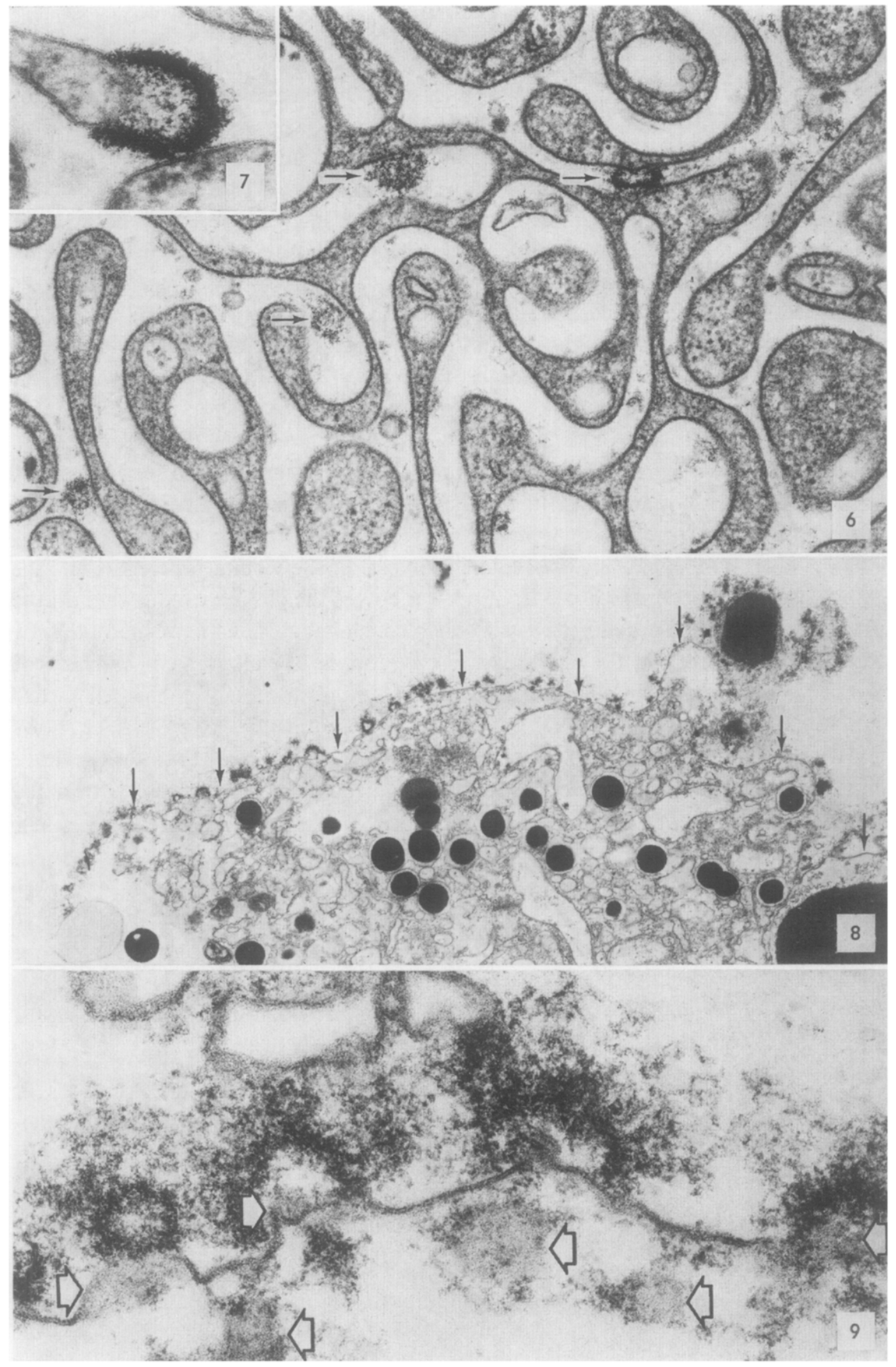


bordered on one side by the debris in the exovate and on the other by alveolar cytoplasm (Figs. 14 and 15). Individual filaments are difficult to resolve. They are not oriented strictly in parallel but take a somewhat wavy course (Fig. 15). Individual filaments measure about $6-7 \mathrm{~nm}$ in diameter (Fig. 17). The region where the filament bundles are observed corresponds to the zone of alveolar cytoplasm containing the dense conglomerates as seen in cross section (Fig. 13). I therefore assume that the conglomerates represent cross-sectioned filament bundles, the $7 \mathrm{~nm}$ particulate elements being cross-sectioned filaments.

Close to the wound the slender surface folds tip over at their distal ends and form partly overlapping lamellae (Figs. 13 and 18). In the zones of close contact the adjacent membrane surfaces remain separated by a gap. The width of the gaps varies and can be less than 4 or $5 \mathrm{~nm}$ at the points of closest approximation (Fig. 20). Fuzzy material adhering to the outer membrane surface is present in the gap (Fig. 21). Besides, condensed patches sandwiched in the junction can also be seen (Fig. 19). No tight junctions nor any other types of specialized cell junction have been observed here.

In the late phase of healing the reduction in size of the wound proceeds while the egg surface slides under the exovate. The latter covers the closing wound area till the surface has sealed. In cross-sectioned wounds conglomerates can be seen in the zone between the exovate and the underlying egg cytoplasm (see also Fig. 11). When the exovate is detached from the surface it leaves behind a densely pigmented "scar" spot which may remain for hours. In electron micrographs the healed surface shows up as a continuous and highly folded surface area. It may protrude somewhat above the remainder of the egg surface. The zone of alveolar cytoplasm expands over the

FIG. 10. Phase contrast micrograph of a cortical wound in an advanced stage of closure. The intact egg surface circumferential to the wound has become rough (arrows). Fixative $B . \times 625$.

FIG. 11. Cortical wound in an advanced stage of healing. Conglomerates of filamentous material (larger arrows) lie in the zone between the exovate $(E O)$ and the heavily pigmented cytoplasm beneath. A multitude of slender surface folds protrude on either side of the exovate. Small arrows: exovate surface layer. Fixative $B . \times 6400$.

FIG. 12. Rough intact surface (see Fig. 10) shows up as a multitude of surface folds. Dense fibrous exudates (arrows). The filamentous layer $(D L)$ embedded in the alveolar cytoplasm is thicker toward the exovate (right). Fixative $B . \times 14800$.

FIG. 13. Marginal zone of the wound shown in Fig. 11 (left part). Alveolated cytoplasm $(A P)$, densely packed filamentous material forming a conglomerate $(C)$, and exovate $(E O)$. Slender surface folds tip over at their distal ends (arrows). Fixative $B . \times 17600$.

FIG. 14. Zone of transition between exovate and intact egg surface, tangential section. Alveolar cytoplasm $(A P)$, filament bundle $(F)$, and exovate $(E O)$. Fixative $B$. $\times 17600$.

FIG. 15. As Fig. 14. The filaments constituting the bundle are fairly well aligned. Individual filaments are difficult to resolve (arrows). Exovate $(E O)$, alveolar cytoplasm $(A P)$. Fixative $B . \times 26100$.

FrG. 16. Bulk of a conglomerate of filamentous material (cross section). It consists of $7 \mathrm{~nm}$ particulate elements (arrows). The dense core surrounded by a lighter halo (right) represents the cener of the conglomerate. Fixative $A . \times 151000$.

FiG. 17. Bundle showing individual $6-7 \mathrm{~nm}$ filaments (arrows). Fixative $A . \times 75500$. 


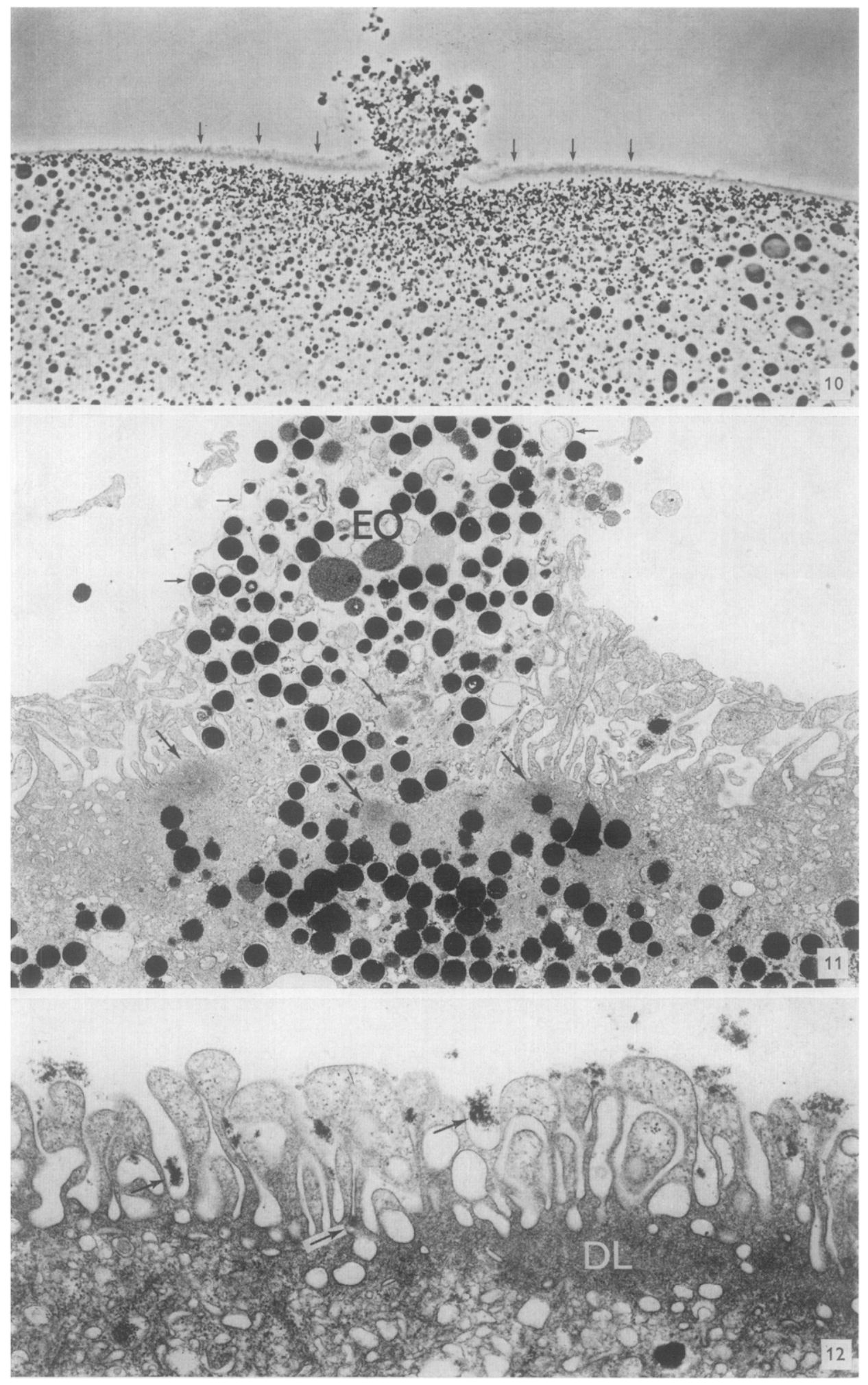




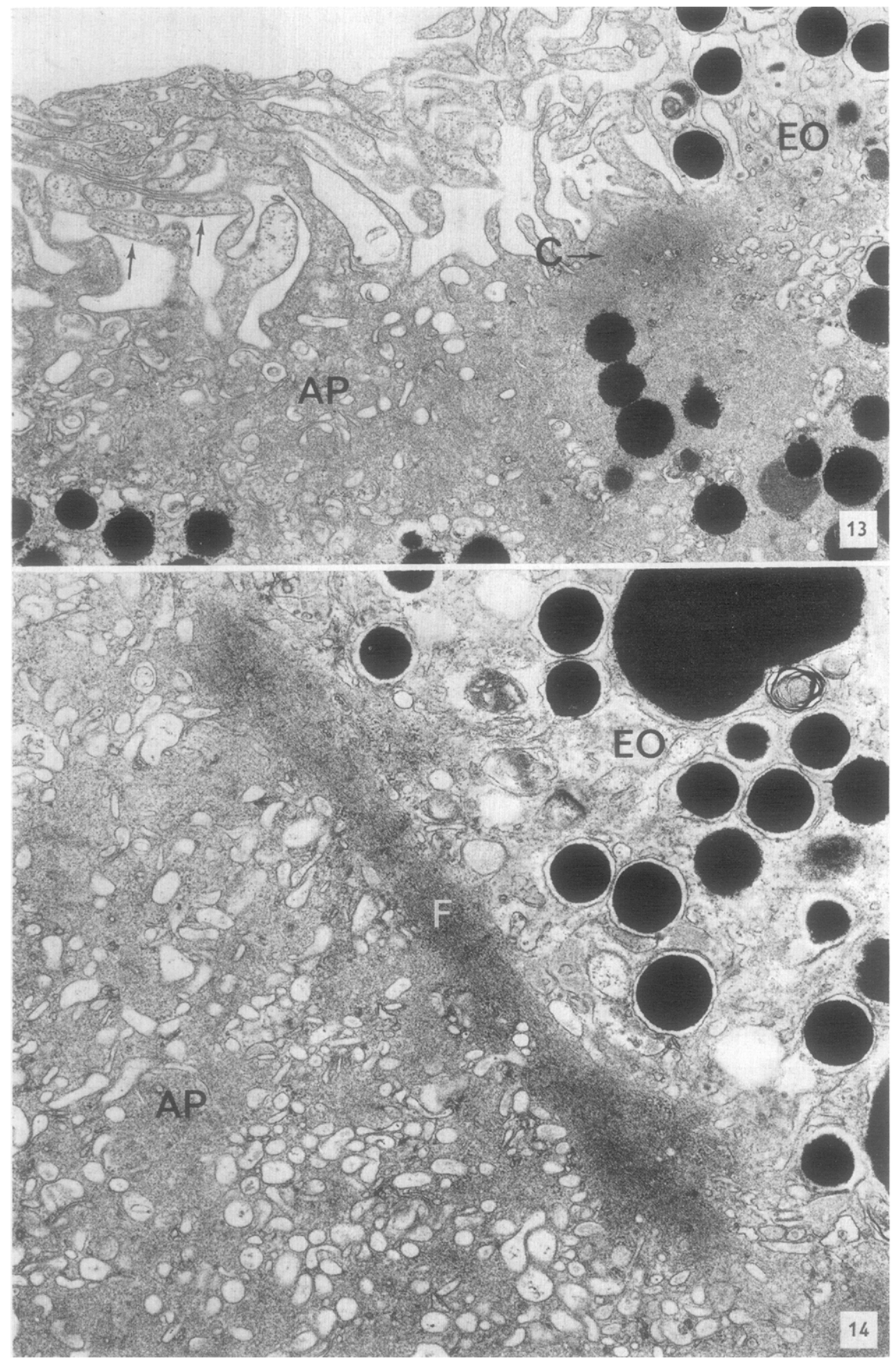




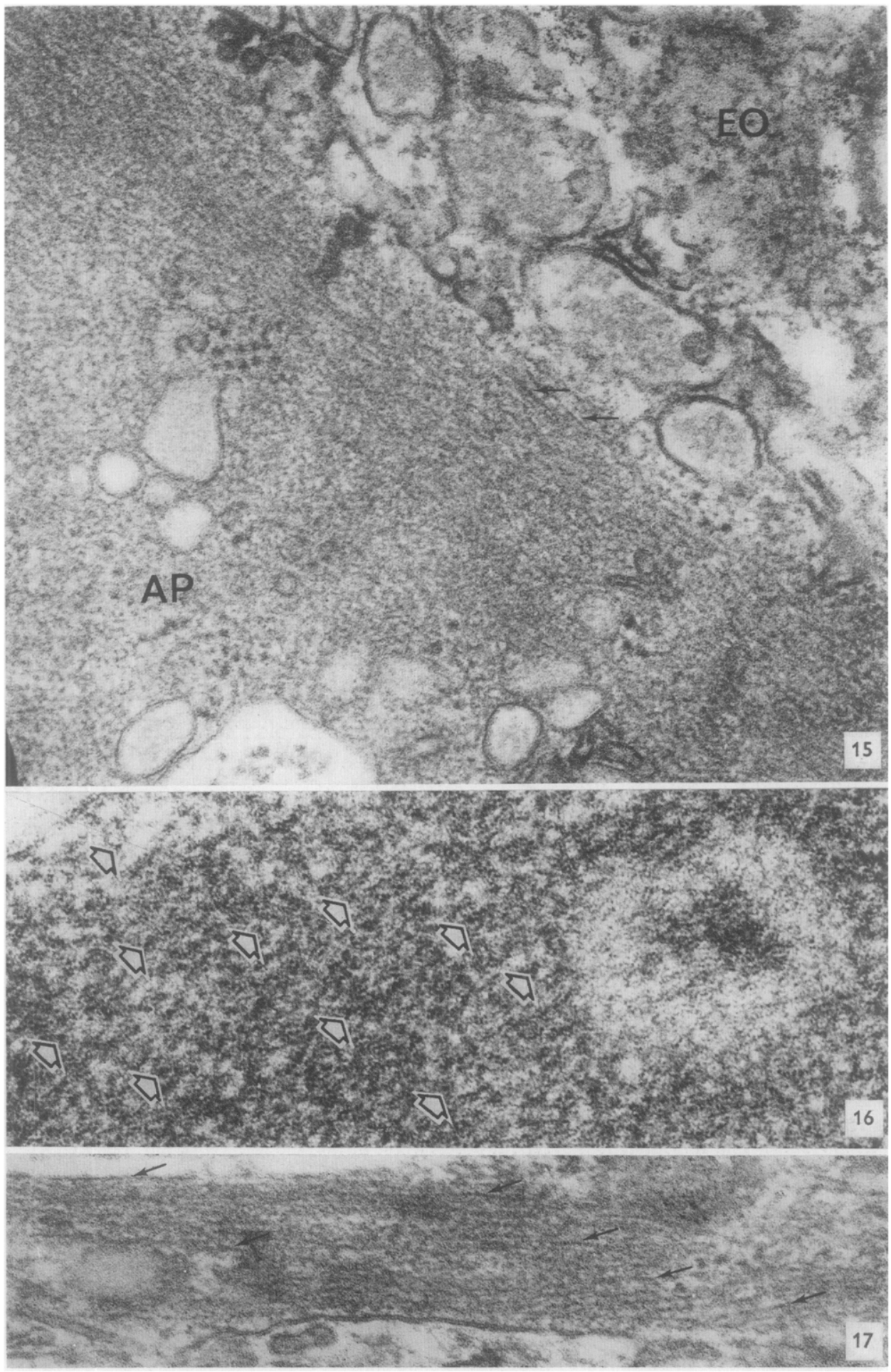


former wound area. Conglomerates of filamentous material lie in this zone in the top part of the bulge.

In material fixed and treated to preserve lipid material following De Bruijn's method, additional structures are visualized. The surface folds next to the exovate contain many dense droplets, most of them adhering closely to the plasma membrane (Figs. 22 and 23). The droplets measure ca. 30-120 nm across. They are not uniform in density but show a substructure of tiny translucent dots (Fig. 23). A multitude of such droplets are interspersed in the debris forming the exovate. The triple-layered membrane covering the surface folds is continuous with a membrane which spans the exovate surface over a considerable distance. Many dense droplets adhere to or are contiguous with the inner side of this membrane (Fig. 24). The droplets are closely similar in size and appearance to the so-called foamy droplets found in wounded amoebae by Szubinska (18).

\section{Additional experimental findings}

It seems plausible to speculate that the filament bundles found represent part of a continuous circular band of filaments that operates like a drawn purse string (13). The following experiment was done to see whether any type of wound will first assume a circular shape before closing. Two long cuts in the form of a cross were made using slightly bent, sharp tungsten needles. As Xenopus eggs will not survive such large wounds, eggs of Pleurodeles waltlii were used for this experiment. These eggs exhibited a much better wound healing capacity in Tris-buffered Holtfreter solution at $\mathrm{pH} 6.3$ (2). One needle was inserted in such a way that the tip lay directly underneath and parallel to the cortex. By lifting the needle slightly the cortex was pulled taut. By rubbing the second needle over the first the cortex could be gently squeezed until it split apart. This resulted in a clean cut, the cortex not being torn free from the underlying cytoplasm. The second cut was made in the same way. The healing process of such a cross-shaped wound is illustrated in Fig. 25. It was found that in the late phase first a ring-shaped wound is formed, which seems to close in the fashion of a drawn purse string.

In another experiment a large tear wound was made. The wound assumed an ovoid outline and the edges started moving inward. Then a second incision was made per-

FIG. 18. Margin of a healed wound cut slightly oblique to the egg surface. The surface folds branch (arrows) and tip over at their distal ends, forming overlapping lamellae. Fixative $B, \times 11300$.

FIGs. 19-21. As Fig. 18. Gap junctions seen at points where membrane surfaces of the folds are closely apposed. Fixative $B . \times 103400$.

FIG. 22. Surface fold. Foamy droplets in contact with the plasma membrane. Fixative C. $\times 63900$. Frg. 23. Foamy droplets contiguous with a triple-layered membrane in the exovate. Arrow points at a typical foamy droplet. Fixative $C . \times 63900$.

FIG. 24. A part of the exovate surface. Dense droplets are contiguous with a triple-layered membrane. The membrane branches irregularly in places (arrows). Fixative $C . \times 103400$. 


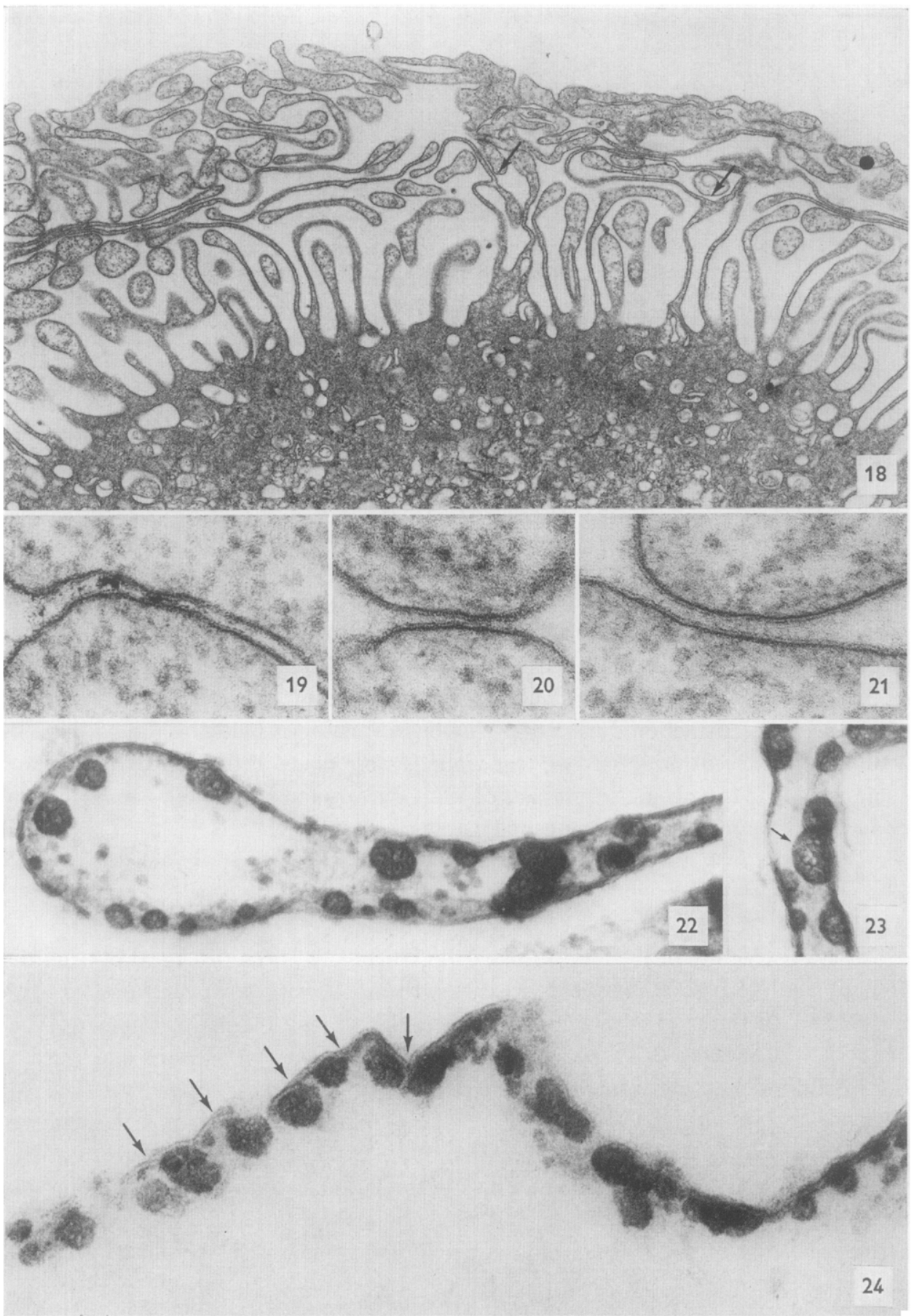




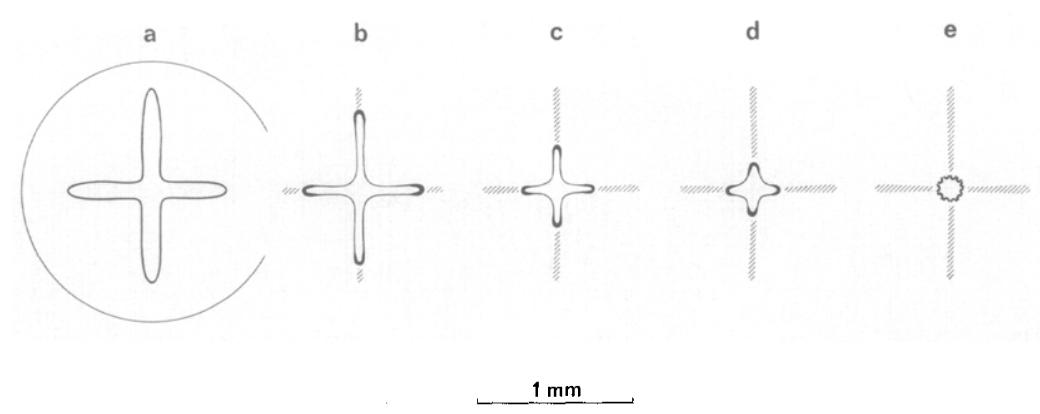

Fig. 25. Healing of a cross-shaped wound in Pleurodeles egg. Shaded area: newly repaired surface. ( $a$ and $b$ ) During the first couple of minutes the edges of the cuts come closer while the slits shorten rapidly. The corners of intact cortex between the arms of the cross retract slightly and round off. (c) Four minutes after wounding. The tips of the arms have retracted markedly. The width of the cuts has remained almost the same. (d) Six minutes after wounding. The wound edge has increased in pigmentation and is starting to assume a circular form. (e) Eight minutes after wounding. Final phase of wound closure, the central open area closes in the fashion of a drawn purse string.

pendicular to the wound edge. The closing movement stopped while the new cut changed into a wide V-shape. As soon as the edges of this cut had moved so far apart that they came in line with the original edge the closing movement continued. The $V$-shaped widening of the second incision shows that the wound edge is under tension.

I have tried to block wound closure by exposing Xenopus eggs to a concentration of 100 or $250 \mu \mathrm{g} / \mathrm{ml}$ vinblastine sulfate (VB). According to my earlier observations (unpublished) contraction during early furrow formation is halted in the presence of VB at these concentrations. Now, tear wounds were made in the constricted part of such a blocked furrow in the presence of VB. Contrary to expectation, wound closure consistently was as perfect as normally.

\section{DISCUSSION}

The ultrastructure of the egg periphery in Xenopus has been discussed in detail by Hebard and Herold (8). The isolated cortex shown here (Fig. 2) comprises the cell membrane, the layer of feltlike material (dense layer), and the peripheral cytoplasm. This $5 \mu \mathrm{m}$ wide layer corresponds to the cortex as defined by Hebard and Herold, but in addition contains some of the small yolk granules and lipid droplets. The isolated cortex structurally conforms to the description of the isolated surface coat as given originally by Holtfreter ( 9 , pp. 262, 263). Discussing the process of cortical wound healing, Holtfreter formulates his interpretation as follows (p. 280): "That the stretching of dye marks or of cells in the circumference of a wound is really due to active spreading of the coat and not, as might be assumed at a first glance, to being 
stretched by an actively constricting wound edge, ..."; (p. 281): "The main question is what are the intimate changes that take place, when the membrane changes from a state of equilibrium, apparently reached at the point of maximal retraction to a motion in opposite sense? Where does the new energy come from which forces the wound edge to spread against the pulling strength of the rest of the membrane?" These questions are still topical.

With regard to wound closure recent EM studies have put much emphasis on the presence of a contractile microfilament system in the cortex $(7,12)$. Also from the micrographs presented in this paper the conclusion seems warranted that microfilaments are involved in the process of healing. The mechanism could be that arrayed filaments form a circular band which by contraction closes the wound. However, I hesitate in drawing this conclusion, first of all because the appearance of filaments in bundles may result from passive accumulation in the retracting wound edges of nonparallel filaments present in the dense layer, and, second, because I found that in the presence of vinblastine sulfate (VB) the contractile mechanism which leads to the appearance of the early furrow is blocked, whereas wound closure is not. According to my own observations (unpublished) 100 or $250 \mu \mathrm{g} / \mathrm{ml} \mathrm{VB}$ prevents the formation of an early furrow, i.e., contraction, when applied 6 minutes before the onset of cleavage (Xenopus laevis eggs without vitelline membrane). The same treatment blocks the progression of the early furrow within 6 minutes when applied at the onset of cleavage. The shallow groove formed by constriction does not proceed further, but very slowly fades away. In electron micrographs a disrupted pattern of filaments can be seen between 16 and 30 minutes after the onset of treatment. Evidence exists that the cortical filaments in amphibian eggs are actinlike (16). Actin isolated from various animal sources is known to precipitate upon binding to VB $(6,21)$. Changes in cell shape upon VB-treament have been related to the disruption of filament organization $(10,11)$. It seems that VB paralyses the contractile system. If both in cleavage (for references see $l$ ) and wound healing active contraction is caused by identical filaments, then it is not clear why wound healing is not blocked also.

The healing of a cross-shaped wound, as illustrated, shows that a purse string-like structure in the strict sense is not a prerequisite for the closure of a wound (cf. 13). The initial narrowing and shortening of the slits are more easily explained in terms of centripetally directed, active surface expansion ( 9 ), but contraction certainly cannot be ruled out. In the other experiment described a second incision was made perpendicular to the wound edge. The experiment shows that the inward moving edge is under tension, which, however, does not necessarily imply that active contraction is taking place. Active contraction or active expansion?-let us consider the latter possibility more closely.

Following De Bruijn's method, foamy droplets can be shown in the surface folds 8-721836 . Ultrastructure Research 
and along the surface of the exovate, as well as inside it. Most droplets are contiguous with a triple-layered membrane (Fig. 24). This membrane differs from the plasma membrane in that it branches irregularly in places. The structure is morphologically similar to that seen during the formation of the so-called "new membrane" in wounded amoebae (18). It is striking that with two dissimilar procedures, in two different types of animal cell, both wounded, an almost identical and so far unique structure is revealed. The difference in the conditions of preservation reduces the likelihood that we are dealing with artifacts. According to Szubinska's concept (18) the foamy droplets represent pools of membrane precursor material. This material is thought upon fusion to contribute to the plasma membrane and to expand its surface area. According to this reasoning the strongly folded surface (Fig. 22) partly represents newly formed membrane.

During the healing process pigmented surface moves from the circumference toward the wound (9). Concomitantly the strongly folded region next to the wound increases in area. According to my interpretation the latter observation also suggests that the spreading is not just a passive smoothing-out of the existing cell surface under a pulling force from the wound margin, although Gingell (7) interprets the extensive folding of this whole region as a manifestation of contraction only. Recalling Holtfreter's last question, see above, surface expansion and the resulting closure of the wound against the tangential forces of the rest of the surface could occur by means of growth of new membrane surface. However, membrane growth in itself does not explain the centripetally directed movement of cytoplasmic inclusions (pigment) of the cortex.

After normal fixation the dense fuzzy component adhering externally to the surface of the folds and of the exovate is visualized. With De Bruijn's method no fuzzy material is seen, but now foamy droplets adhering to the inner surface are visualized (Fig. 24). With this knowledge in mind vaguely visible droplets can be detected beneath the crenelated layer also after normal fixation (Fig. 9). Therefore I believe the crenelated layer to be identical with the membrane with associated droplets. This structure may reflect that a distinct layer is formed on the surface of the exovate. This layer may be functionally similar to the new membrane seen in wounded amoebae (18).

Semicircular lumps of fuzzy material identical to those shown here have been found in the first-cleavage furrow of Xenopus (1). In the furrow they form part of a lanthanum-staining surface layer which is thought to function in interlinking the blastomeres. In cortical wounds the dense fuzzy exudate may also have a sealing function. During coagulation it may act as a glue assisting in forming a coherent mass out of the extruded cytoplasm, while during the final sealing process it may agglutinate the laterally apposed surfaces of the folds (gap junctions). The crenelated layer certainly does not become an integral part of the repaired surface; during the final phase 
of wound closure it is detached together with the exovate. Hence it may be considered as a protective cover of a temporary nature.

Owing to the great irregularity of the surface profile, it was not possible to ascertain whether or not the plasma membrane had reestablished continuity at the end of the healing process.

In further experiments the following suggestions would be worth testing:

1. In tear wounds filaments assemble along the edge during retraction. Insofar as they remain in contact with the underlying cytoplasm, they aggregate into a coherent system building up enough tension to counteract further tangential retraction.

2. Growth of new cell surface circumferential to the wound allows the surface to expand.

3. When new membrane is inserted, the contractile system which has been assembled along the wound edge is uncoupled from the counterbalancing tangential surface forces. It contracts, actively or passively, forming the leading edge of the closing wound.

4. A distinct layer is formed on the surface of the exovate. Together with the coagulated cytoplasm of the exovate itself it temporarily seals off the open cytoplasmic area from the external milieu.

5. Definitive sealing occurs by lateral apposition (i.e., agglutination) of surface folds over the wound area.

Summarizing: every experimental treatment which prevents healing may do so by affecting the coherence of the filament system, the formation of new membrane, or the apposition of membrane surfaces (i.e., sealing).

I thank Mr E. van Voorst for technical assistance, and Miss Eva Bartová, Mrs E. G. Aleven. and $\mathrm{Mr} \mathrm{L}$. Boom for preparing the prints and the drawing. I want to thank Professor P. D. Nieuwkoop for his careful reading of the manuscript and Dr J. Faber for editorial assistance.

\section{REFERENCES}

1. BluemInk, J. G., Z. Zellforsch. Mikrosk. Anat. 121, 102 (1971).

2. CuRTis, A. S. G., J. Embryol. Exp. Morphol. 10, 410 (1962).

3. DAwID, J. B., J. Mol. Biol. 12, 581 (1965).

4. De Bruijn, W. C., Doctoral Thesis, Rotterdam, 1969.

5. Elbers, P. J., Ververgaert, P. H. J. Th. and Demei, R., J. Cell Biol. 23, 23 (1965).

6. FINE, R. E. and BRAy, D., Nature (London) New Biol. 234, 115 (1971).

7. Gingell, D., J. Embryol. Exp. Morphol. 23, 583 (1970).

8. Hebard, C. N. and Herold, R. C., Exp. Cell Res. 46, 553 (1967).

9. HolTfReter, J., J. Exp. Zool.93, 251 (1943).

10. JACOB, H., Amsden, Th. and White, J., Proc. Nat. Acad. Sci. U.S. 60, 471 (1972). 
11. Karfunkel, P., Develop. Biol. 25, 30 (1971).

12. LocKWOOD, W. R., Anat. Rec. 150, 129 (1964).

13. LuCkenbili, L. M., Exp. Cell. Res. 66, 263 (1971).

14. McGee-Russell, S. M. and De Bruijn, W. C., in McGee, S. M. and Ross, K. F. A. (Eds.), Cell Structure and Its Interpretation, p. 115. Arnold, London, 1968.

15. NIU, M. C. and TwitTy, V. C., Proc. Nat. Acad. Sci. U.S. 39, 985 (1953).

16. Perry, M. M., John, H. A. and Thomas, N. S. T., Exp. Cell Res. 65, 249 (1971).

17. Stockem, W., Mikroskopie 26, 185 (1970).

18. SzubinsKa, B., J. Cell Biol. 49, 747 (1971).

19. Van Gansen, P., J. Embryol. Exp. Morphol. 15, 365 (1966).

20. Venable, J. H. and Coggeshall, R. A., J. Cell Biol. 42, 407 (1969).

21. Wilson, L., Bryan, J., Ruby, A. and Mazia, D., Proc. Nat. Acad. Sci. U.S. 66, 807 (1970). 